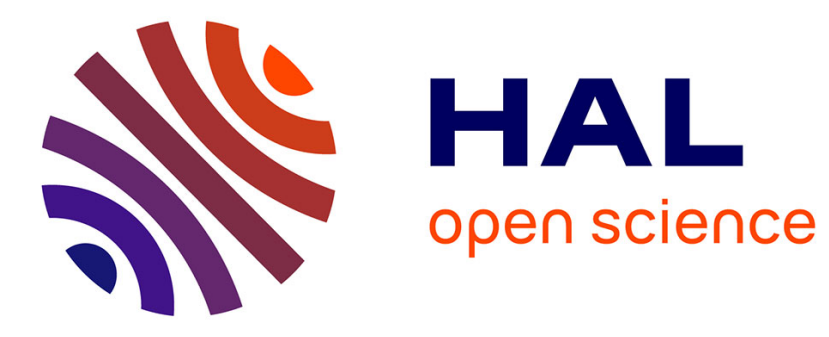

\title{
On the Reliability of Wireless Sensor Networks Communications
}

Alexandre Mouradian, Isabelle Augé-Blum

\section{To cite this version:}

Alexandre Mouradian, Isabelle Augé-Blum. On the Reliability of Wireless Sensor Networks Communications. ADHOC-NOW 2013, Jul 2013, Wroclaw, Poland. p.38-49. hal-00918595

\section{HAL Id: hal-00918595 \\ https://hal.science/hal-00918595}

Submitted on 13 Dec 2013

HAL is a multi-disciplinary open access archive for the deposit and dissemination of scientific research documents, whether they are published or not. The documents may come from teaching and research institutions in France or abroad, or from public or private research centers.
L'archive ouverte pluridisciplinaire HAL, est destinée au dépôt et à la diffusion de documents scientifiques de niveau recherche, publiés ou non, émanant des établissements d'enseignement et de recherche français ou étrangers, des laboratoires publics ou privés. 


\title{
On the Reliability of Wireless Sensor Networks Communications *
}

\author{
Alexandre Mouradian and Isabelle Augé-Blum \\ Université de Lyon, INRIA, INSA Lyon, CITI, F-69621, France \\ firstname.lastname@insa-lyon.fr
}

\begin{abstract}
More and more Wireless Sensor Networks (WSNs) applications and protocols are proposed. Notably, critical applications, which must meet time and reliability requirements. Works on the real-time capability of WSNs have been proposed [1]. In this paper we propose to study the achievable reliability of WSNs, tacking into account the probabilistic nature of the radio link. We define the reliability of a WSN to be the probability that an end-to-end communication is successful (i.e. the packet is received by the sink). We propose a theoretical framework inspired by a reference model [5]. We use the framework to derive the reliability of two types of routing schemes: unicast-based and broadcastbased. We show that in the case of broadcast-based, the sink is a reliability bottleneck of the network. We also discuss the impact of the MAC scheme on the reliability.
\end{abstract}

\section{Introduction}

Wireless Sensor Networks (WSNs) are multihop large scale networks composed of up to thousands of sensor nodes. They are usually deployed to monitor environment parameters of an area, or to monitor some equipments (such as power meters or gas meters). Sensor nodes run on batteries so they should consume as little energy as possible in order to increase the network lifetime. Because WSNs can contain lots of nodes, the financial cost of a node should be as low as possible, this leads to design nodes with poor capabilities (computation, radio, memory, etc...). For these reasons, research on WSNs mainly focused on self-organization and energy consumption efficiency.

Nevertheless, new applications appear. Notably critical applications on which human life and environment may depend. For example forest fire detection application must send an alarm to the sink when a forest fire is detected. Such applications require the respect of time constraints and a high reliability. In our example, the fire alarm must reach the sink before a known time bound and the probability that it reaches the sink must be high. This leads to ask which constraints can be handled by WSNs. Part of the answer is given by previous work [1], which derives a bound on real-time capacity of WSNs. However, this

\footnotetext{
* This work has been partially founded by French Agence Nationale de la Recherche
} under contract VERSO 2009-017. 
work does not take into account the probabilistic nature of the radio link. A protocol that can meet end-to-end deadlines loses its value if its delivery ratio is very low. The goal of this paper is to give an insight on what reliability is achievable in WSNs. We provide a theoretical framework used to model the reliability of WSNs. This framework is inspired form previous works [5] [10]. In this paper, we define the reliability to be the probability that a packet is received by the sink after $H$ hops. The main contributions of this paper are the theoretical framework and its application, with different sets of hypotheses, to derive the achievable reliability of WSNs. Notably with a link reliability that depends on the emitter-receiver distance and a broadcast-based routing scheme.

In section 2 we describe related works. In section 3 we present our theoretical framework. In section 4 and 5 we apply the framework, respectively with a basic and realistic propagation model. In section 6, we discuss the impact of MAC on reliability results. In section 7 , we conclude and give future works.

\section{Related work}

In the literature, some reliability models for WSNs have been proposed with different definitions of reliability. The authors of [2] define the reliability of a WSN to be the probability to have a minimum rate of information delivered to the sink. They propose an algorithm in order to compute it. Nevertheless their approach is not focused on failures coming from the probabilistic radio link but more on node failures. In [9], the authors present a theoretical framework to compute the reliability of transport protocols in WSNs. This framework uses an elegant block diagram approach and includes several possible faults. Nevertheless, this is a high level model which not able to capture the complexity of realistic communications in WSNs. In [3] authors study the reliability of multipath routing in ad-hoc networks. They take into account the probabilistic radio link, but the link quality is not dependent on the emitter-receiver distance (it is a parameter of the model) and they derive reliability equation for peer-to-peer traffic, not convergecast as in WSNs. Our proposition is complementary to these approaches. It focuses on the reliability of the end-to-end communications by modeling physical, MAC and routing layers.

Several reliable communication protocols for WSNs have been proposed [13][11] [12][7]. Our framework allows to understand better why these communication protocols are reliable and also to develop new, and even more reliable, schemes.

\section{Theoretical framework}

\subsection{Unreliable links}

Usually, the signal transmitted through the wireless channel is not only attenuated by the distance between the emitter and receiver, but it also experiences random attenuation coming from changes in the environment (moving objects, etc). Due to this random attenuation, the reception of a packet is probabilistic, 
i.e. there is a probability that a transmission between two nodes fails. In the remainder of this paper we will consider two link models: a basic model in which a node has a predefined set of neighbor and a probability that a packet sent is correctly received by a neighbor, and a more realistic model in which the node can potentially communicate with every node of the network with a probability that depends on the emitter-receiver distance.

In the basic model, each node is provided with a set of neighbor nodes which it can communicate with. Nevertheless the communications between a node and its neighbors are unreliable. Indeed, a neighbor of a transmitting node can receive the packet with a probability $P_{b c r}$. In this basic model we assume that the probability is the same for all neighbor nodes.

The realistic model is based on the log-normal propagation model. In [14], the authors advocate that it provides a realistic propagation model for WSNs. In this case, the reception probability depends on the emitter-receiver distance (at constant transmission power). The probability to receive correctly a packet is a function: $P_{c r}(d)$ with $d$ the emitter-receiver distance. More information on this model can be found in [4]. It is possible to consider packet retransmissions in the probability formula as in [6].

We can notice that, in both models, we consider the probability for a node to receive a packet is independent from the probability for another node.

\section{$3.2 \quad$ Network topology}

Our network model is based on [5] and [10]. $N$ nodes are placed randomly and uniformly on a disk of area 1 . The sink is placed at the center of the disk. Messages are generated by nodes in the network and must be routed to the sink in a multihop fashion. We assume that the nodes are distributed uniformly on the disk, thus on average there is one node in a $\frac{1}{N}$ surface. We can also determine the radius of a disk that contains $d$ nodes on average:

$$
R=\sqrt{\frac{d}{\pi N}}
$$

We also assume that a gradient has been constructed in the network. As in [13], a node is given a number that corresponds to the number of hops a packet originated from this node has to do to reach the sink. In the case of the basic model, the number of hops a packet has to do depends on the size of the neighbors set of a node which is linked to the nodes range by equation 1 . In the case of unreliable links, the hop-count is difficult to evaluate because any node can potentially communicate with the sink or with any other nodes (with a given probability). We thus assume that the gradient is constructed using a threshold. Above a given probability of reception threshold two nodes are considered to be neighbors. Since we assumed the nodes are uniformly distributed, the nodes with the same hop-count form concentric rings of width $R$ centered on the sink, as depicted on the left-hand side of Fig. 1 (In the case of realistic model, the ring width depends on the probability threshold). 


\subsection{Protocols}

In this work, we focus more on the influence of routing on reliability because we are interested in end-to-end reliability. Nevertheless the impact of MAC operation is discussed in section 6. We divide MAC schemes into two categories regarding reliability, in the first, the MAC mechanism ensure that the packet is received (no collisions or resolved collisions). In the second, the packet may not be correctly received (unresolved collisions).

We use the number of hops as a routing metric. The shortest path is the path composed of the less possible number of hops according to the gradient. When the gradient is constructed, several metrics can be used (probability of reception, energy,...). Thus many routing metrics can be mimiced by the number of hops metric, by carefully constructing the gradient. We present two ways of routing which lead to different reliability as it is shown in the remainder of this paper.

In unicast-based routing, a shortest path from the source to the sink is selected (using the gradient). A node sends the packet to the neighbor which is next in the path. In the remainder of the paper we derive the reliability formulas of the presented schemes.

With a broadcast-based scheme, the packet is broadcasted to the neighbors of a node (as in [13] for example). A set of potential forwarders compete to relay the packet. In our case it is the nodes with a smaller hop-count (known thanks to the gradient presented in section 3.2). The selection of the forwarder can be made based on several criteria (signal strength, energy level, random, etc). In this paper we do not detail this process and we assume that a unique node is arbitrarily chosen among all potential forwarders which have correctly received the packet.

\section{Case 1: the basic radio link model}

In this section we derive the end-to-end communication probability from the presented theoretical framework with the basic propagation model. Here we assume that the first MAC scheme is used, i.e. no packet is lost due to MAC operation.

\subsection{Unicast scheme}

We consider that the routing scheme selects a path from the sender to the sink. A node belonging to the path forward the packet to the neighbor that is next in the path. The probability that one hop is successful is $P_{b c r}$. The probability that the packet is correctly received by the sink after $H$ hops is given by equation 2 .

$$
P_{e 2 e_{-} 1 u}=\prod_{i=0}^{H} P_{b c r}=\left(P_{b c r}\right)^{H}
$$

The number of hops depends on the range of a node:

$$
H=\left\lceil\frac{1}{R \sqrt{\pi}}\right\rceil
$$


$\frac{1}{\sqrt{\pi}}$ is the radius of the disk of area 1 . The probability $P_{e 2 e_{-} 1 u}$ goes to zero when $R$ goes to zero (because $H$ goes to $+\infty$ and $0 \leq P_{b c} \leq 1$ ) and it is equal to $P_{b c}$ when $R \geq \frac{1}{\sqrt{\pi}}$. It means that, in the case of the basic model, when there are less hops, the communications are more reliable. This is true because $P_{b c r}$ does not decrease in function of the emitter-receiver distance. The basic model fails to capture the fact that long communications are less reliable. Nevertheless it is still useful when the difference between the furthest neighbor and the closest neighbor of a node is small.

Using a large value for $R$ is not only a problem because long range communications are unreliable (notice that increasing the power of transmission increases the reliability) but also because it reduces the possible spatial reuse (simultaneous communications in the network) and thus reduce the achievable throughput as mentioned in [5].

\subsection{Broadcast scheme}

In the case of broadcast-based routing, there is not only one forwarder but there is a set of potential forwarders. This set is defined by the routing protocol used. In our case, one of the shortest paths must be selected, the set of potential forwarders is thus the nodes with a smaller hop count than the sender.

With the gradient information we can define the set of forwarders by determining the percentage of the neighbors of a node which have smaller hop-count. If no gradient information is provided this percentage has to be determined according to the routing scheme used.

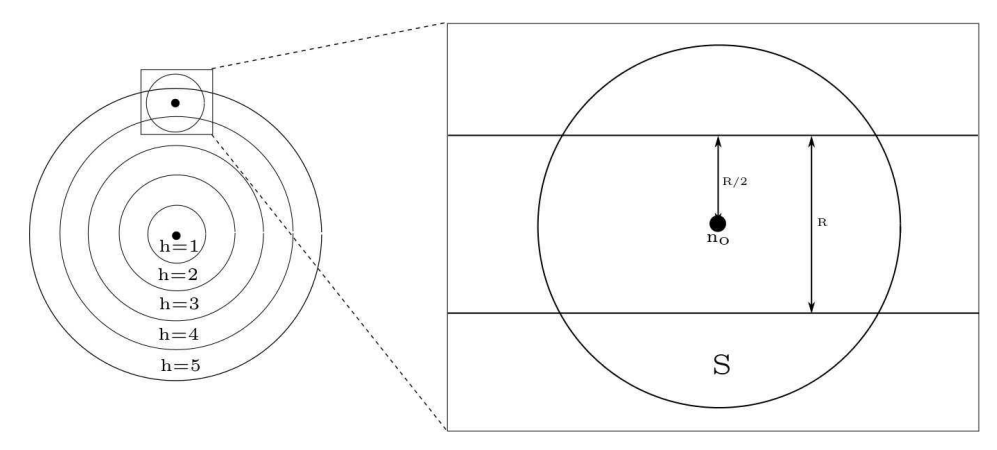

Fig. 1. Surface S

As shown on Fig. 1, we assume that if a ring $h$ has a sufficiently large radius, it can be considered as two parallel straight lines at the scale of a node. This is not true for rings close to the sink, thus a correction factor might have to be considered. For exact calculation, see [8]. With this assumption, a node is on average placed at equal distance from the ring edges. It means it has, on average, 
an overlapping surface $S$ with the lower ring. The surface $\mathrm{S}$ is the surface of a segment:

$$
S=\left(\frac{\pi}{3}-\frac{\sqrt{3}}{4}\right) R^{2}
$$

The ratio between the surface of the disk defined by the range of the node and the surface $S$ is given by equation 5 .

$$
r=\frac{S}{\pi R^{2}}=\left(\frac{1}{3}-\frac{\sqrt{3}}{4 \pi}\right)
$$

We know there are $d$ nodes in the range of the node and, as nodes are distributed uniformly, the number of nodes in an area is proportional to the surface of this area. We thus define $m$ the number of potential forwarders to be: $m=r d$.

The probability that one hop is successful, in the case of broadcast-based routing, is the probability that at least one potential forwarder receives the packet. The probability that the end-to-end communication is successful is the probability that every hop is successful. It is given by equation 6 for a $H$ hops path.

$$
\begin{aligned}
P_{e 2 e_{-} 1 b} & =P_{b c r} \prod_{h=1}^{H-1}\left(1-\prod_{j=1}^{m}\left(1-P_{b c r}\right)\right) \\
& =P_{b c r}\left(1-\left(1-P_{b c r}\right)^{m}\right)^{H-1}
\end{aligned}
$$

For the last hop the only potential forwarder is the sink so $h$ ranges from 1 to $H-1$. The last hop is thus less reliable because if the sink fails to receive the packet, it is completely lost as in the case of unicast-based routing. The sink can thus be seen as a reliability bottleneck. We can notice that the number of potential forwarders $m$ should be smaller for rings closer to the sink, because these rings are smaller. In order to take this effect into account the surface $S$ should have to be computed using the technique from [8] where the exact surface formula is given. Then $m$ would be a function of $h$.

When $m$ goes to $+\infty,\left(1-P_{b c r}\right)^{m}$ goes to 0 thus $P_{e 2 e_{-} 1 b}$ goes to $P_{b c r}$. It means that the more potential forwarder there is, the more reliable the end-toend communication is. But, the sink is a reliability bottleneck so $P_{e 2 e_{-} 1 b}$ does not go to 1 when $m$ goes to $+\infty$. It means that by putting several sinks instead of one at the center of the disk area we can increase the reliability. Similarly to the unicast case, the reliability increases also when there are less hops. Nevertheless same issues as unicast case, about long range communications and spatial reuse appear.

Broadcast-based routing is more reliable than the unicast-based scheme. We do not give a formal proof here, but intuitively, we observe that in the case of broadcast-based scheme, to have a successful communication we need that, at 
least, one node receives the packet. So if all communications but one fails, the communication is successful. In the case of unicast, if the unique communication fails the packet is lost. On the other hand, broadcast-based routing involves more nodes. The potential forwarders have to be awake and wait for packets. A mechanism for forwarder election has to be implemented in order to select the forwarder. These mechanisms consume energy. This highlight a trade-off between energy consumption and reliability.

\section{Case 2: the realistic radio link model}

In this section, we use a more realistic propagation model in order to derive the end-to-end communication probability. In this case, the probability that a packet is correctly received depends on the emitter-receiver distance.

\subsection{Unicast scheme}

With the unicast-based routing scheme, a path from a node to the sink is predefined. Nevertheless in the case of the realistic model, every node in the network can potentially communicate with every other node (in some cases this probability is very low). So two cases are possible, either the forwarder of a packet sent by a node in the path must be the next hop in the path, or it can be any node of the path closer to the sink, as in [7].

In the former case, a packet originated from a node in ring $H$ has to travel a distance of $H \times R$ by doing $H$ hops. The probability to reach the sink is thus obtained by substituting $P_{c r}(R)$ for $P_{b c r}$ in equation 2 . Here the construction of the gradient is important. Indeed, if the probability threshold (mentioned in section 3 ) is low the probability to receive correctly the packet for one hop is low but a hop has a longer distance. On the contrary if probability threshold is high the probability for one hop is high but a hop has a shorter distance. This latter alternative is better for reliability (for example if a node is three hops away from the sink with $P_{c r}(R)=0.95$ then $P_{e 2 e}=0.86$, with another gradient threshold it is six hops away from the sink and $P_{c r}(R)=0.99$ then $\left.P_{e 2 e}=0.94\right)$ but more hops lead more transmissions and thus to higher end-to-end delays.

In the latter case, every node on the path closer to the sink than the sender can relay the packet. We assume that a node forwards a packet it has just received only if no node closer to the sink has received it (only nodes belonging to the path can receive the packet). We can notice that it is not a strictly unicast solution because there are more than one receiver. A protocol which implements this behavior is provided in [7]. For a packet originated in ring $H$, there are $H-1$ potential forwarders (plus the sink). For each potential forwarder, the probability that it is elected is equal to the probability that it receives the packet and that no node closer to the sink receives it. The end-to-end communication probability is the sum of the probabilities that each node of the path is a forwarder and the probability to reach the sink from the forwarder. The probability to reach the sink from the forwarder is defined similarly to the end-to-end communication, 
but with $H$ being the ring of the forwarder. The end-to-end communication probability is thus a recursive function defined by equations 7 and 8 .

$$
\begin{gathered}
P_{e 2 e \_2 u}(0)=1 \\
P_{e 2 e \_2 u}(H)=\sum_{h=0}^{H-1}\left\{\prod_{j=0}^{h-1}\left[1-P_{c r}((H-j) R)\right] \times P_{c r}((H-h) R) \times P_{e 2 e \_2 u}(h)\right\}
\end{gathered}
$$

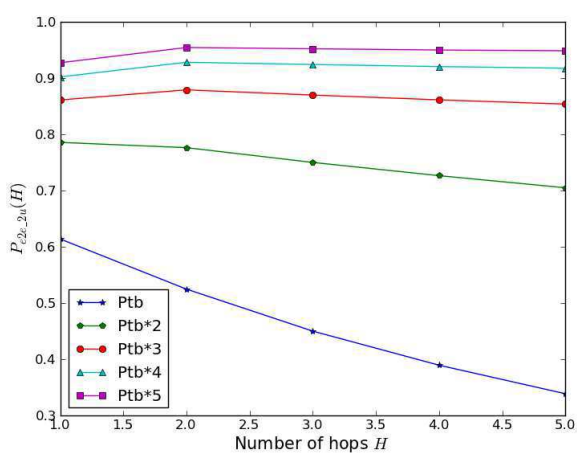

Fig. 2. $P_{e 2 e_{-} 2 u}$ in function of $H$ for different transmission powers

Fig. 2 is a plot of equation 8 with $H$ ranging from 1 to 5 and different transmission powers (the transmission power here depends on a basis value $P_{t b}$ ). We observe that, for the lower transmission powers, the probability of a successful end-to-end communication decreases when $H$ increases. For higher transmission powers, probability can be higher for $H=2$ than for $H=1$. This is due to the fact that the probability to communicate from the ring $H=2$ to the sink is high when the transmission power increases. The reliability in this case is higher than in the strictly unicast case (intuitively a packet has more options to reach the sink). Nevertheless, the former solution requires that all nodes of the path closer to the sink than the sender are awake at each hop. This increases energy consumption.

\subsection{Broadcast scheme}

With this scheme, a node can relay a packet if it is closer to the sink than the sender and no node closer to the sink receives the packet. But unlike the unicast second case, in this case, there are several potential forwarders in each ring $h$. We thus have to determine the number of potential forwarders at a given ring, the number of nodes closer to the sink and the distance of those nodes from 
the sender (because the probability of reception depends on the emitter-receiver distance).

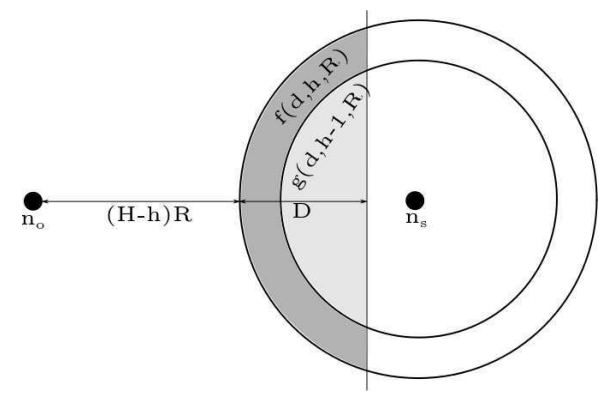

Fig. 3. Link between surfaces and $D$

The number of nodes in ring $h$ noted $m_{h}$ and the number of nodes closer to the sink noted $m_{h-}$ are given respectively by equations 9 and 10

$$
\begin{gathered}
m_{h}=\left\lfloor\left(\pi(R h)^{2}-\pi[R(h-1)]^{2}\right) N\right\rfloor \\
m_{h-}=\left\lfloor\left(\pi[R(h-1)]^{2}\right) N\right\rfloor
\end{gathered}
$$

In order to compute the probabilities, we need to evaluate the distance between the sender and the potential forwarders. Since nodes are uniformly distributed on the disk, the number of nodes in an area is proportional to the size of the area. The range of a node defines a disk area. Thus the first neighbor of a node is at least at a distance that allows the two nodes to be in the same circle. In our case we define areas as depicted on Fig. 3. The segments areas $f$ and $g$ are defined in function of the distance $D$, so the emitter-receiver distance is linked to the surface and the surface is linked to the number of nodes. We can thus determine the distance between the sender and the $n^{t h}$ node in the ring $h$ (notice that in this case again the circle corresponding to the range of node $n_{o}$ is approximated by a straight line).

The function $f$ is the surface of segment of ring $h$, and is defined as follows:

- if $0<D<R$ :

$$
f(D, h, R)=\cos ^{-1}\left(1-\frac{D}{h R}\right)(h R)^{2}-(R h-D) \sqrt{2 h R D-D^{2}}
$$

- if $R \leq D \leq(2 h-1) R$ :

$$
\begin{aligned}
f(D, h, R)= & \cos ^{-1}\left(1-\frac{D}{h R}\right)(h R)^{2}-(R h-D) \sqrt{2 h R D-D^{2}} \\
& -\cos ^{-1}\left(1-\frac{D-R}{(h-1) R}\right)((h-1) R)^{2}
\end{aligned}
$$




$$
+(R(h-1)-D+R) \sqrt{2(h-1) R(D-R)-(D-R)^{2}}
$$

- if $D \geq(2 h-1) R$ :

$$
\begin{aligned}
f(D, h, R)= & \cos ^{-1}\left(1-\frac{D}{h R}\right)(h R)^{2}-(R h-D) \sqrt{2 h R D-D^{2}} \\
& -\cos ^{-1}\left(1-\frac{D-R}{(h-1) R}\right)((h-1) R)^{2}
\end{aligned}
$$

The inverse function with respect to $\mathrm{D}$ gives the distance in function of the surface (with $S=n / N$ for the $n^{\text {th }}$ neighbor):

$$
f^{-1}(S, h, R)=D
$$

Similarly, the surface for nodes closer to the sink is given by equation 15 :

$$
g(D, h, R)=\cos ^{-1}\left(1-\frac{D}{h R}\right)(h R)^{2}-(R h-D) \sqrt{2 h R D-D^{2}}
$$

and $g^{-1}(S, h, R)$ is defined similarly to $f^{-1}$.

As in the second case of the unicast scheme, the probability of a successful end-to-end communication is a recursive function defined by equations 16 and 17 .

$$
\begin{gathered}
P_{e 2 e_{2} 2 b}(0)=1 \\
P_{e 2 e \_2 b}(H)=\sum_{h=0}^{H-1}\left\{\left[1-\prod_{j=1}^{m_{h}}\left(1-P_{c r}\left(f^{-1}\left(\frac{j}{N}, h, R\right)+R \times(H-h)\right)\right)\right]\right. \\
\times\left[\prod_{k=1}^{m_{h}-}\left(1-P_{c r}\left(g^{-1}\left(\frac{k}{N}, h-1, R\right)+R \times(H-(h-1))\right)\right)\right] \\
\left.\times P_{e 2 e \_2 b}(h)\right\}
\end{gathered}
$$

The probability that an end-to-end communication, originated at ring $H$, is successful is the sum of the probabilities for each ring that at least one node of the ring $h$ receive the packet, and no node closer to the sink receives the packet, and the probability to reach the sink from ring $h$.

We can notice that when $h=0, f^{-1}$ and $g^{-1}$ are equal to $0, m_{h}$ is equal to 1 (the sink node) and $m_{h-}$ is equal to 0 (similarly, for $h=1, m_{h-}$ is equal to 1 and $g^{-1}$ is equal to 0$)$.

Fig. 4(a) is the plot of equation 17 with $N$ ranging from 50 to 2000 and $H$ from 1 to 5 with a power transmission of $P_{t b} \times 2$. The probability of a successful end-to-end communication increases with $N$ up to a maximal value that depends on $H$. The maximal value is less than one. By increasing the number of nodes $N$, the number of potential forwarder in any ring increases but there is still only 


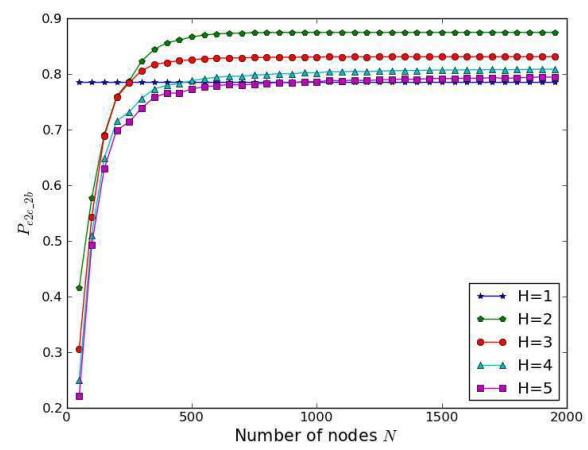

(a) $H$ varies and $P_{t b} \times 2$

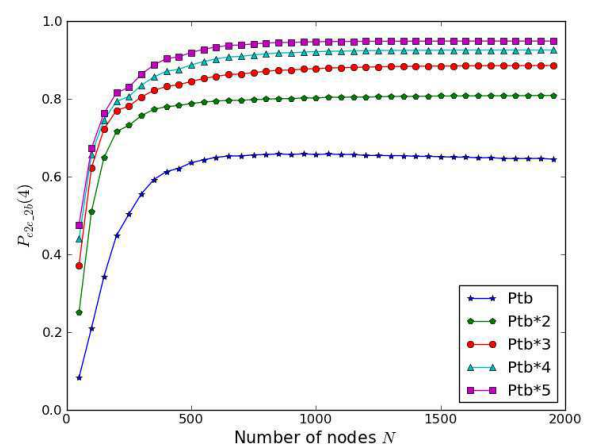

(b) Transmission power varies and $H=4$

Fig. 4. $P_{e 2 e_{-} 2 b}$ in function of $N$

one sink so the probability cannot converge to one. As in the case of the basic model, the sink can be seen as the reliability bottleneck of the network.

Fig. 4(b) is the plot of equation 17 with $N$ ranging from 50 to 2000 and the transmission power from $1 \times P_{t b}$ to $5 \times P_{t b}$ with $H=4$. In this case, the probability also reaches a maximal value. This maximal value increases with the transmission power because communications are more reliable. We can remark that, by adding sinks (enlarging the bottleneck), we improve the reliability without increasing the transmission power, thus without spending more energy (indeed new sinks consume energy but it does not reduce the network lifetime).

\section{Impact of MAC}

In the previous sections we consider that no packet is lost due to MAC operation. The probability to lose a packet only comes from the radio propagation. In this section, we consider that the probability to receive a packet is the probability that the packet is not lost because of the MAC (unresolved collision) and that it is not lost due to the propagation.

In the case of the unicast-based routing and the basic model the only change is that the probability $P_{b c r}$ in equation 2 is replaced by $P_{b c r_{-} n o d e t}=P_{b c r} \times P_{n o \_c o l l}$

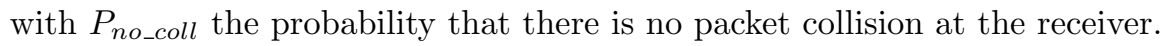

In the case of broadcast-based routing and the basic model, if there is a collision, it may prevent several potential forwarders from receiving the packet. In the worst case, all the potential forwarders cannot decode the packet because of the collision. In this case, equation 6 becomes:

$$
P_{e 2 e_{1} 1 b}=\left(P_{b c r} \times P_{n o \_c o l l}\right) \times\left(\left(1-\left(1-P_{b c r}\right)^{m}\right) \times P_{n o \_c o l l}\right)^{H-1}
$$

The broadcast-based scheme reliability is thus highly reduced by MAC unresolved collisions. Similarly, these results can be easily extended to the realistic propagation model case with the same conclusions. 


\section{Conclusion and future works}

In this paper we develop a theoretical framework which aims at studying the reliability of WSNs. We apply it to two types of propagation models with different routing and MAC schemes. Our results show that the broadcast solutions are more reliable than unicast based ones. Nevertheless, this comes at a price in terms of energy consumption and throughput. We also conclude that the sink is a reliability bottleneck in the case of the broadcast-based scheme. The presented framework allows a better understanding of the reliability of end-to-end communications in WSNs and thus guide protocol designers' decisions in order to meet applications reliability requirements.

More information on WSNs reliability can be derived from our model, for example we plan to further investigate the effect of the gradient construction probability threshold on the reliability. We also plan to enhance our model by taking into account correlations between reception probabilities of different nodes of the same area. The influence of MAC and routing acknowledgment packet shall also be considered.

\section{References}

1. Abdelzaher, T., Prabh, S., Kiran, R.: On Real-Time Capacity Limits of Multihop Wireless Sensor Networks. pp. 359-370. RTSS'04, Lisbon, Portugal (2004)

2. AboElFotoh, H.M., ElMallah, E.S., Hassanein, H.S.: On the reliability of wireless sensor networks. In: IEEE ICC'06. pp. 3455-3460 (2006)

3. Caleffi, M., Ferraiuolo, G., Paura, L.: A reliability-based framework for multi-path routing analysis in mobile ad-hoc networks. International Journal of Communication Networks and Distributed Systems 1(4), 507-523 (2008)

4. Goldsmith, A.: Wireless Communications. Cambridge University Press (2005)

5. Gupta, P., Kumar, P.: The capacity of wireless networks. IEEE Transactions on Information Theory 46(2), 388-404 (2000)

6. Jaffrès-Runser, K.: Worst case delay analysis for a wireless point-to-point transmission. In: RTN'12 (colocated with ECRTS'12). Pisa, Italy (2012)

7. Lampin, Q., Barthel, D., Augé-Blum, I., Valois, F.: Qos oriented opportunistic routing protocol for wireless sensor networks. In: Wireless Days. pp. 1-6 (2012)

8. Mouradian, A., Augé-Blum, I.: 1-d coordinate based on local information for mac and routing issues in wsns. pp. 42-55. ADHOC-NOW'12 (2012)

9. Shaikh, F.K., Khelil, A., Suri, N.: On modeling the reliability of data transport in wireless sensor networks. In: IEEE PDP'07. pp. 395-402 (2007)

10. Watteyne, T.: Energy-efficient slef-organization for wireless sensor networks. Phd thesis manuscript (2008)

11. Yang, F., Augé-Blum, I.: Constructing virtual coordinate for routing in wireless sensor networks under unreliable links. In: IWCMC. pp. 815-819 (2009)

12. Yang, F., Augé-Blum, I.: Delivery ratio-maximized wakeup scheduling for ultra-low duty-cycled wsns under real-time constraints. Com. Netw. 55(3), 497-513 (2011)

13. Ye, F., Zhong, G., Lu, S., Zhang, L.: Gradient broadcast: a robust data delivery protocol for large scale sensor networks. Wirel. Netw. 11, 285-298 (May 2005)

14. Zuniga, M., Krishnamachari, B.: Analyzing the transitional region in low power wireless links. In: IEEE SECON'04. pp. 517-526 (2004) 